\title{
Distributed Cooperative Control of Multiple Nonholonomic Mobile Robots
}

\author{
Gang Wang • Chaoli Wang • Qinghui Du $\cdot$ Lin Li • \\ Wenjie Dong
}

Received: 11 June 2015 / Accepted: 11 November 2015 / Published online: 14 January 2016

(C) The Author(s) 2016. This article is published with open access at Springerlink.com

\begin{abstract}
In this paper, the distributed cooperative control problem is considered for multiple type $(1,2)$ nonholonomic mobile robots. Firstly, a local change of coordinates and feedback is proposed to transform the original nonholonomic system to a new transformed system. Secondly, a distributed controller for the transformed system is designed by using information of the intrinsic system and its neighbors to make the state converge to the same value asymptotically. Furthermore, it shows that the same value can be confined to the origin, which means that the problem of cooperatively converging to a stationary point of a group of nonholonomic systems can be practically
\end{abstract}

G. Wang · C. Wang $(\bowtie) \cdot$ L. Li

University of Shanghai for Science and Technology,

Shanghai 200093, China

e-mail: clclwang@126.com

G. Wang

e-mail: 2010wanggang@gmail.com

L. $\mathrm{Li}$

e-mail: lilin0211@163.com

Q. Du

Department of Mathematics, Luoyang Normal University, Luoyang 471022, China

e-mail: meihao2526@163.com

\section{W. Dong}

Department of Electrical Engineering, The University of Texas Rio Grande Valley, Edinburg, TX 78539, USA

e-mail: wenjie.dong@utrgv.edu solved. Finally, due to the communication delays are inevitable in practice, new distributed controllers for the transformed system are also proposed making the state converge to the same value or zero asymptotically with considering communication delays. The proposed methods are then extended to the case where the nonholonomic mobile robot needs to form a prescribed formation other than agreeing on a same value. The stability of the proposed methods is proved rigorously. Simulation results confirm the effectiveness of the proposed methods.

Keywords Distributed control · Nonholonomic mobile robots · Formation control - Cooperative control

\section{Introduction}

In recent years, there has been an increasing research interest in the distributed synchronization control of multi-agent systems due to its potential applications in many areas, such as formation control [1, 2], design of distributed sensor networks [3], flocking control [4, $5]$, etc. Some seminal works are $[6,7]$, just to name a few.

A large number of effective control approaches have focused on two control problems of networked systems, i.e., leaderless consensus problems and leader-following consensus problems. For leaderless 
consensus problems, controllers are designed to drive all the agents to a common value, which depends on initial conditions (see $[8,9]$ ). As for leader-following consensus problems, controllers are designed to make all the follower nodes track the trajectory of the leader node (see [10, 11]). Besides, there are also many works investigated for different types of agent dynamics including first-order integrator systems [12, 13], second-order integrator systems $[14,15]$ and higherorder integrator systems [16, 17]. However, many practical cooperative control applications involve agents that are nonlinear and nonholonomic. The stabilization problem of nonholonomic system cannot be solved by many methods of classical linear system for the fact nonholonomic system fails to meet the three necessary conditions of the theorem of Brockett [18]. Thus the above mentioned methods cannot the solve the cooperative control of multiple nonholonomic agents. To solve the single nonholonomic system control problem, many scholars have done a lot of relevant research in this area (see [19-23], etc.). But most of the methods focused on the single nonholonomic system cannot solve the cooperative control of multiple nonholonomic systems directly, because we consider multiple nonholonomic mobile robots and the associated controller is distributed in nature-for each robot has access to the state of its neighbors only. Motivated by those observations, the authors in [2, 24-28] have focused on the cooperative control of multiple nonholonomic agents. In [2], Lin, Francis, and Maggiore have studied the feasibility problem of achieving a specified formation among a group of nonholonomic unicycles by local distributed control. In [24], Dong and Farrell presented two controllers for cooperative control problems of nonholonomic systems. One distributed controller was proposed to make a group of nonholonomic mobile agents cooperatively converge to some stationary point; The other controller was proposed to make a group of mobile agents converge to and track a target point which moves along a desired trajectory under various communication scenarios. And they also extended the methods to solve the problem of cooperative control of multiple nonholonomic dynamic systems with uncertainty in [25]. In [26], Liu and Jiang proposed a new class of distributed nonlinear controller for leader-following formation control of unicycle robots by using nonlinear small-gain design methods. In [27],
Dong studied the distributed tracking control of multiple nonholonomic chained systems. Different from their works in $[24,25]$, the assumption that all follower robots have access to the information of the leader robot is not needed. In other words, for each robot, the available information for feedback is its own information and its neighbours' information. In [28], Cao, Jiang, and Yue have also investigated the consensus problems of multiple nonholonomic systems. Distributed controller was constructed by using the theory of cascaded systems. Different to previous assumptions on the group reference such as persistent excitation or converging to nonzero constant in [24], the condition on the group reference signal has been further relaxed.

Campion, Basin, and D'Andréa-Novel claimed that the interesting nonholonomic wheeled mobile robots are type $(2,0),(2,1),(1,1),(1,2)$ robots in [29]. In this paper, we study distributed cooperative control problem of multiple type $(1,2)$ nonholonomic mobile robots. This kind of systems is more complicated, compared with type $(2,0)$, type $(2,1)$ and type $(1,1)$. The idea exploited in this paper can be used to investigate the same problem of the other three nonholonomic wheeled mobile robots. The main contributions of this paper are threefold. First, a local change of coordinates and feedback is proposed to transform the original nonholonomic system to a new transformed system. Second, distributed controllers for the new transformed system are designed by using its own information and its neighbours' information to make the state converge to the same value or zero asymptotically with and without considering communication delays. Third, extension is provided to extend the proposed schemes to the case, where the nonholonomic mobile robot needs to form a stable formation other than agreeing on a same value.

The remainder of this paper is organized as follows. In Section 2, some notions and preliminaries about the algebraic graph theory are briefly introduced, and the kinematic of type $(1,2)$ and the distributed cooperative control problem of type $(1,2)$ are presented. In Section 3, under two different communication scenarios, distributed controllers are designed to ensure that the state of each transformed system converges to the same value or zero asymptotically. Extensions are provided in Section 4. In Section 5, the simulation results are shown to illustrate the performance of the 
proposed methods. Some conclusions are given in the last Section.

\section{Problem Statement}

\subsection{Basic Graph Theory and Notations}

In this subsection, some notions and preliminaries about the algebraic graph theory are briefly introduced.

Let $\mathcal{G}=\{\mathcal{V}, \mathcal{E}\}$ denote a directed graph, where $\mathcal{V}=$ $\{1, \ldots, N\}$ is the set of nodes corresponding to each robot, and $\mathcal{E} \subseteq \mathcal{V} \times \mathcal{V}$ is the set of edges. $(i, j) \in \mathcal{E}$ means that robot $j$ can obtain information from robot $i$, but not necessarily vice versa for a directed graph. In this paper, self-loop is not allowed in the graph, that is, $(i, i) \notin \mathcal{E} . N_{i}=\{j \in \mathcal{V} \mid(j, i) \in \mathcal{E}\}$ denotes the neighbors of robot $i$. A matrix $A=\left[a_{i j}\right] \in R^{N \times N}$ denotes the adjacency matrix of $\mathcal{G}$, where $a_{i j}>0$ iff $(j, i) \in \mathcal{E}$, else $a_{i j}=0$. It is assumed that the topology is fixed which means $A$ is time-invariant. A matrix $L=D-A$ is called the Laplacian matrix of $\mathcal{G}$, where $D=\operatorname{diag}\left(d_{1}, \ldots, d_{N}\right)$ is the in-degree matrix with $d_{i}=\sum_{j=1}^{N} a_{i j}$. A direct path from robot $i$ to robot $j$ is a sequence of successive edges in the form $\{(i, l),(l, m), \ldots,(k, j)\}$. Graph $\mathcal{G}$ is strongly connected if any two robots $(i, j)$ with $i \neq j$, there is a direct path from robot $i$ to robot $j$. A directed graph $\mathcal{G}$ has a spanning tree, if there exists a robot $i$ such that there is a direct path from robot $i$ to every other robot in the graph, where the robot $i$ is called the root of graph $\mathcal{G}$. A directed graph $\mathcal{G}$ is balanced if $\underline{1}^{T} L=0$, where $\underline{1}$ is a vector with element one. Bidirectional graph is a special case of a directed graph, if $(i, j) \in \mathcal{E}$, then $(j, i) \in \mathcal{E}$. Meanwhile, it is stipulated that $a_{i j}=a_{j i}$ in bidirectional graph.

\subsection{Kinematic of the Mobile Robots}

Consider a group of $N(N \geq 2)$ type $(1,2)$ nonholonomic mobile robots as shown in Fig. 1 Each robot has two steering wheels (conventional centered orientable wheels) and one castor wheel (conventional off-centered orientable wheel). $\left(x_{i}, y_{i}\right)$ denotes the position $P_{i}$ of the center of the $i$ th $(i=1,2, \ldots, N)$ robot's mass, $\theta_{i}$ denotes the angle between $x_{i 1}$-axis and $X$-axis, and $\beta_{i 1}$ and $\beta_{i 2}$ denote angles between

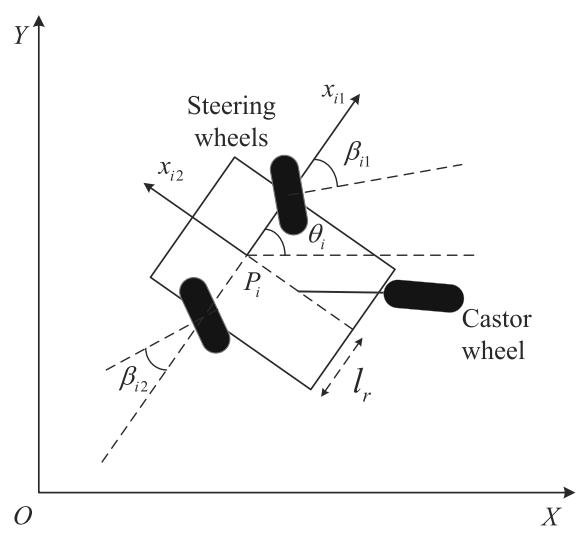

Fig. 1 Type $(1,2)$ nonholonomic mobile robot

the orientation of the plane of steering wheels and $x_{i 1}$-axis, $l_{r}(>0)$ is half of the width of the $i$ th robot. The nonholonomic constraint of the $i$ th robot is defined by [29]

$\left\{\begin{array}{l}\left(\cos \beta_{i 1}, \sin \beta_{i 1}, l_{r} \sin \beta_{i 1}\right) H\left(\theta_{i}\right) \dot{\xi}_{i}=0, \\ \left(-\cos \beta_{i 2},-\sin \beta_{i 2}, l_{r} \sin \beta_{i 2}\right) H\left(\theta_{i}\right) \dot{\xi}_{i}=0,\end{array}\right.$

where $\xi_{i}=\left(x_{i}, y_{i}, \theta_{i}\right)$, and

$H\left(\theta_{i}\right)=\left[\begin{array}{ccc}\cos \theta_{i} & \sin \theta_{i} & 0 \\ -\sin \theta_{i} & \cos \theta_{i} & 0 \\ 0 & 0 & 1\end{array}\right]$.

In addition, Eq. 1 can be specifically written as

$\left\{\begin{array}{l}\dot{x_{i}}=-l_{r} v_{i 1}\left[\sin \beta_{i 1} \sin \left(\theta_{i}+\beta_{i 2}\right)+\sin \beta_{i 2} \sin \left(\theta_{i}+\beta_{i 1}\right)\right], \\ \dot{y_{i}}=l_{r} v_{i 1}\left[\sin \beta_{i 1} \cos \left(\theta_{i}+\beta_{i 2}\right)+\sin \beta_{i 2} \cos \left(\theta_{i}+\beta_{i 1}\right)\right], \\ \dot{\theta}_{i}=v_{i 1} \sin \left(\beta_{i 2}-\beta_{i 1}\right), \quad \dot{\beta}_{i 1}=v_{i 2}, \quad \dot{\beta}_{i 2}=v_{i 3},\end{array}\right.$

where $q_{i}=\left[x_{i}, y_{i}, \theta_{i}, \beta_{i 1}, \beta_{i 2}\right]^{T}$ is the state of the $i$ th robot, and $v_{i 1}, v_{i 2}, v_{i 3}$ are the velocity of castor wheel and two angular velocities of steering wheels of the $i$ th robot, respectively.

\subsection{Cooperative Control Problem}

The chained form systems were first introduced in [30] as a class of systems to which one could convert a number of interesting examples, and for which it was easy to derive steering control laws. However, only the systems that have two input and one chain were focused on. In our manuscript, the type $(1,2)$ nonholo- 
nomic mobile robot has three inputs and two chains. Thus, the state feedback and coordinate transformation proposed in [30] cannot be utilized directly. The sufficient conditions for converting a multiple-input and multiple-chain system with nonholonomic constraints into a chained form via state feedback and a coordinate transformation were presented in $[31,32]$. Here, we invoke the coordinate and state transformation which is similar to that in [32]. Then, to simplify the distributed cooperative controller design, a novel change of states by adding $\int_{0}^{t} \omega(s) d s$ based on chained form is proposed as follows.

$$
\begin{aligned}
z_{i 1}= & \theta_{i}-\int_{0}^{t} \omega(s) d s, \\
z_{i 2}= & x_{i} \cos \theta_{i}+y_{i} \sin \theta_{i}, \\
z_{i 3}= & x_{i} \sin \theta_{i}-y_{i} \cos \theta_{i}, \\
z_{i 4}= & -x_{i} \sin \theta_{i}+y_{i} \cos \theta_{i}-2 l_{r} \frac{\sin \beta_{i 1} \sin \beta_{i 2}}{\sin \left(\beta_{i 2}-\beta_{i 1}\right)} \\
& +\gamma_{1} \omega\left(x_{i} \cos \theta_{i}+y_{i} \sin \theta_{i}\right), \\
z_{i 5}= & x_{i} \cos \theta_{i}+y_{i} \sin \theta_{i}-l_{r} \frac{\sin \left(\beta_{i 1}+\beta_{i 2}\right)}{\sin \left(\beta_{i 2}-\beta_{i 1}\right)} \\
& +\gamma_{2} \omega\left(x_{i} \sin \theta_{i}-y_{i} \cos \theta_{i}\right), \\
u_{i 1}= & v_{i 1} \sin \left(\beta_{i 2}-\beta_{i 1}\right), \\
u_{i 2}= & -v_{i 1} \sin \left(\beta_{i 2}-\beta_{i 1}\right)\left(x_{i} \cos \theta_{i}+y_{i} \sin \theta_{i}\right) \\
& +2 l_{r} v_{i 3} \frac{\sin ^{2} \beta_{i 1}}{\sin ^{2}\left(\beta_{i 2}-\beta_{i 1}\right)} \\
& -2 l_{r} v_{i 2} \frac{\sin ^{2} \beta_{i 2}}{\sin ^{2}\left(\beta_{i 2}-\beta_{i 1}\right)}+l_{r} v_{i 1} \sin \left(\beta_{i 1}+\beta_{i 2}\right), \\
u_{i 3}= & v_{i 1} \sin \left(\beta_{i 2}-\beta_{i 1}\right)\left(x_{i} \sin \theta_{i}-y_{i} \cos \theta_{i}\right) \\
& +l_{r} v_{i 3} \frac{\sin ^{2}\left(2 \beta_{i 1}\right)}{\sin ^{2}\left(\beta_{i 2}-\beta_{i 1}\right)} \\
& -l_{r} v_{i 2} \frac{\sin ^{2}\left(2 \beta_{i 2}\right)}{\sin ^{2}\left(\beta_{i 2}-\beta_{i 1}\right)}+2 l_{r} v_{i 1} \sin \beta_{i 1} \sin \beta_{i 2},
\end{aligned}
$$

where $\omega=\rho \sin t$, and $\rho, \gamma_{1}, \gamma_{2}$ are positive constants.

Taking derivative of Eq. 3, we have

$$
\begin{aligned}
& \dot{z}_{i 1}=u_{i 1}-\omega, \\
& \dot{z}_{i 2}=-\gamma_{1} z_{i 2} \omega^{2}+\omega z_{i 4}+\left(u_{i 1}-\omega\right)\left(z_{i 4}-\gamma_{1} \omega z_{i 2}\right), \\
& \dot{z}_{i 3}=-\gamma_{2} z_{i 3} \omega^{2}+\omega z_{i 5}+\left(u_{i 1}-\omega\right)\left(z_{i 5}-\gamma_{2} \omega z_{i 3}\right), \\
& \dot{z}_{i 4}=u_{i 2}+\gamma_{1} \dot{\omega} z_{i 2}+\gamma_{1} \omega u_{i 1} z_{i 4}-\gamma_{1}^{2} \omega^{2} u_{i 1} z_{i 2}, \\
& \dot{z}_{i 5}=u_{i 3}+\gamma_{2} \dot{\omega} z_{i 3}+\gamma_{2} \omega u_{i 1} z_{i 5}-\gamma_{2}^{2} \omega^{2} u_{i 1} z_{i 3} .
\end{aligned}
$$

Remark 1 It should be noted that because of the local nature of the state and feedback transformations (3), the laws designed for the transformed system (4) do not guarantee global stability properties for the original model (2) of the $i$ th type $(1,2)$ nonholonomic mobile robot. Indeed, since the coordinate transformation and state feedback are well defined over the subset $\Omega_{i}=\left\{\left(x_{i}, y_{i}, \theta_{i}, \beta_{i 1}, \beta_{i 2}\right) \in R^{5} \mid \beta_{i 1} \neq\right.$ $\left.\beta_{i 2} \bmod \pi\right\}$. We have that only within such a domain can we obtain "global" stability.

Definition 1 The distributed cooperative control problem of multiple type $(1,2)$ nonholonomic mobile robots (2) discussed in this paper is to design the distributed control input $u_{i}=\left[u_{i 1}, u_{i 2}, u_{i 3}\right]^{T}$ for the $i$ th system (4) using $z_{i}=\left[z_{i 1}, z_{i 2}, z_{i 3}, z_{i 4}, z_{i 5}\right]^{T}$ and the relative state $z_{l}$ of its neighbors for $l \in N_{i}$ such that $z_{i}$ is bounded and $\lim _{t \rightarrow \infty}\left(z_{i}(t)-z_{j}(t)\right)=0$ for $1 \leq i \neq j \leq N$.

Remark 2 The control laws are required to make the state $z_{i}$ of each transformed system converge to the same value $c(t)$ with $c(t)=\left[c_{1}, c_{2}(t), c_{3}(t), c_{4}, c_{5}\right]^{T}$, where $c_{1}, c_{4}$, and $c_{5}$ are constants which are unknown and depend on robots' initial conditions and communication between robots, and $c_{2}(t), c_{3}(t)$ are bounded functions. Furthermore, if $\lim _{t \rightarrow \infty}\left(u_{i 1}(t)-\omega(t)\right)=0$, $c_{1}=0, c_{4}=0$, and $c_{5}=0$, then $c_{2}=0, c_{3}=0$ (see Lemma 2). Since the system (2) discussed in this paper is nonholonomic, by the theorem of Brockett [18], the state $q_{i}$ of each original system (2) cannot be stabilized at a stationary point by a smooth pure state feedback controller which is a smooth function of its own state $q_{i}$ and the states $q_{l}$ of its neighbors for $l \in N_{i}$. To overcome this difficulty, we design cooperative control laws such that the state $z_{i}$ of each transformed system (4) converges to a moving vector $c(t)$. Then, we will state that $c(t)$ can also be confined to the origin, which means that cooperatively converging to a stationary point of a group of nonholonomic systems (2) can be practically solved. For details, please refer to the remarks after Theorem 2.

An additional assumption on the communication topology is given below.

Assumption 1 The communication digraph $\mathcal{G}$ has a spanning tree and $\mathcal{G}$ with weight matrix $A$ is balanced.

Remark 3 Note that this assumption is very common which has appeared in relevant literature such as Dong [33]. And it is much more relaxed than undirected connected graph as has been made in Hou, Cheng, and Tan [8], Ou, Du, and Li [34], Feng and Wen [35]. 
The following lemmas are useful in our design and analysis of distributed controllers.

Lemma 1 (Dong and Farrell [24]) If the digraph $\mathcal{G}$ has a spanning tree and the Laplacian matrix $L$ of the digraph $\mathcal{G}$ with weight matrix $A=\left[a_{i j}\right]\left(a_{i j} \geq 0\right)$, then

$\lim _{t \rightarrow \infty} e^{\mu t}\left(e^{-L t}-\underline{1} w^{T}\right)=0$

for any $\mu \in\left[0, \operatorname{Re}\left(\lambda_{2}(L)\right)\right)$, where $\lambda_{2}$ is the nonzero eigenvalue of $L$ with the smallest real part, $w$ satisfies $w^{T} L=0$ and $w^{T} \underline{1}=1$.

Lemma 2 (Dong [33]) If the digraph $\mathcal{G}$ has a spanning tree and the Laplacian matrix $L$ of the digraph $\mathcal{G}$ with weight matrix $A$ is balanced, the matrix $L^{T}+L$ is semidefinite. Furthermore, if $\lim _{t \rightarrow \infty} x^{T}\left(L^{T}+L\right) x=$ 0 for a vector $x=\left[x_{1}, x_{2}, \ldots, x_{N}\right]^{T}$, then

$\lim _{t \rightarrow \infty}\left(x_{i}(t)-x_{j}(t)\right)=0,1 \leq i \neq j \leq N$.

Before proceeding further, the following additional lemma is required.

Lemma 3 For the ith transformed system (4), if $u_{i 1}-$ $\omega, z_{i 4}, z_{i 5}$ are bounded and converge to zero asymptotically, then $z_{i 2}, z_{i 3}$ are bounded and converge to zero asymptotically.

$V_{1}=\frac{1}{2}\left(z_{i 2}^{2}+z_{i 3}^{2}\right)$.

Differentiating $V_{1}$ along with solutions of system (4), we get

$$
\begin{aligned}
\dot{V}_{1}= & -\omega^{2}\left(\gamma_{1} z_{i 2}^{2}+\gamma_{2} z_{i 3}^{2}\right)+\omega z_{i 2} z_{i 4}+\omega z_{i 3} z_{i 5} \\
& +z_{i 2}\left(u_{i 1}-\omega\right)\left(z_{i 4}-\gamma_{1} \omega z_{i 2}\right) \\
& +z_{i 3}\left(u_{i 1}-\omega\right)\left(z_{i 5}-\gamma_{2} \omega z_{i 3}\right) \\
\leq & -2 \underline{\gamma} \omega^{2} V_{1}+2 \varphi_{1} V_{1}+2 \varphi_{2} \sqrt{V_{1}}
\end{aligned}
$$

where $\underline{\gamma}=\min \left\{\gamma_{1}, \gamma_{2}\right\}$, and

$\varphi_{1}=\bar{\gamma}|\omega|\left|u_{i 1}-\omega\right|, \varphi_{2}=\frac{1}{\sqrt{2}}\left(\left|z_{i 4}\right|+\left|z_{i 5}\right|\right)\left|u_{i 1}\right|$,

with $\bar{\gamma}=\max \left\{\gamma_{1}, \gamma_{2}\right\}$.
Due to boundedness of $\omega$, and $\lim _{t \rightarrow \infty}\left(u_{i 1}\right.$ $\omega)=0, \lim _{t \rightarrow \infty} z_{i 4}(t), z_{i 5}(t)=0$, we have $\lim _{t \rightarrow \infty} \varphi_{1}(t), \varphi_{2}(t)=0$. In order to facilitate the following analysis, we take $\sigma=\sqrt{V_{1}}$, then $D^{+} \sigma \leq$ $-\gamma \omega^{2} \sigma+\varphi_{1} \sigma+\varphi_{2}$, where $D^{+}$is the upper Dini derivative. Thus, we get

$$
\begin{aligned}
\sigma(t) \leq & e^{\int_{0}^{t}\left(-\underline{\gamma} \omega^{2}(s)+\varphi_{1}(s)\right) d s} \sigma(0) \\
& +\int_{0}^{t} e^{\int_{\tau}^{t}\left(-\underline{\gamma} \omega^{2}(s)+\varphi_{1}(s)\right) d s} \varphi_{2}(\tau) d \tau
\end{aligned}
$$

Note that

$$
\begin{aligned}
\int_{0}^{t}-\underline{\gamma} \omega^{2}(s) d s & =\int_{0}^{t}-\underline{\gamma} \rho^{2} \sin ^{2} s d s \\
& =-\underline{\gamma} \rho^{2}\left(\frac{t}{2}-\frac{\sin 2 t}{4}\right) .
\end{aligned}
$$

With this observation in mind, since $\lim _{t \rightarrow \infty} \varphi_{1}(t)=0$, there always exists $T_{1}>0$ such that $\varphi_{1}(t) \leq \frac{\gamma \rho^{2}}{4}$ for all $t \geq T_{1}$. Define function $\bar{\varphi}_{1}(t)=\sup _{0 \leq \tau \leq t} \varphi_{1}(\tau)$, the following equation can be achieved

$$
\begin{aligned}
\int_{0}^{t} \varphi_{1}(s) d s & =\int_{0}^{T_{1}} \varphi_{1}(s) d s+\int_{T_{1}}^{t} \varphi_{1}(s) d s \\
& \leq \bar{\varphi}_{1}\left(T_{1}\right) T_{1}+\frac{\underline{\gamma} \rho^{2}}{4}\left(t-T_{1}\right) .
\end{aligned}
$$

Thus

$$
\begin{aligned}
& \int_{0}^{t}\left(-\underline{\gamma} \omega^{2}(s)+\varphi_{1}(s)\right) d s \\
& \leq-\underline{\gamma} \rho^{2}\left(\frac{t}{2}-\frac{\sin 2 t}{4}\right)+\bar{\varphi}_{1}\left(T_{1}\right) T_{1}+\frac{\underline{\gamma} \rho^{2}}{4}\left(t-T_{1}\right) \\
& \leq-\frac{\underline{\gamma} \rho^{2}}{4} t+\frac{\underline{\gamma} \rho^{2}}{4}+\bar{\varphi}_{1}\left(T_{1}\right) T_{1}-\frac{\underline{\gamma} \rho^{2}}{4} T_{1} \text {. }
\end{aligned}
$$

Hence

$$
\begin{gathered}
\lim _{t \rightarrow \infty} e^{\int_{0}^{t}\left(-\underline{\gamma} \omega^{2}(s)+\varphi_{1}(s)\right) d s} \sigma(0) \\
=e^{\lim _{t \rightarrow \infty} \int_{0}^{t}\left(-\underline{\gamma} \omega^{2}(s)+\varphi_{1}(s)\right) d s} \sigma(0)=0 .
\end{gathered}
$$

Next, we will show that

$$
\lim _{t \rightarrow \infty} \int_{0}^{t} e^{\int_{\tau}^{t}\left(-\underline{\gamma} \omega^{2}(s)+\varphi_{1}(s)\right) d s} \varphi_{2}(\tau) d \tau=0 .
$$


Due to $\lim _{t \rightarrow \infty} \varphi_{2}(t)=0, \forall \eta>0$, we can always find that $T_{2} \geq T_{1}$ such that $\varphi_{2}(t) \leq \eta$ for all $t \geq T_{2}$. Define function $\bar{\varphi}_{2}(t)=\sup _{0 \leq \tau \leq t} \varphi_{2}(\tau)$, we get

$$
\begin{aligned}
& \int_{0}^{t} e^{\int_{\tau}^{t}\left(-\underline{\gamma} \omega^{2}(s)+\varphi_{1}(s)\right) d s} \varphi_{2}(\tau) d \tau \\
= & \int_{0}^{T_{2}} e^{\int_{\tau}^{t}\left(-\underline{\gamma} \omega^{2}(s)+\varphi_{1}(s)\right) d s} \varphi_{2}(\tau) d \tau \\
& +\int_{T_{2}}^{t} e^{\int_{\tau}^{t}\left(-\underline{\gamma} \omega^{2}(s)+\varphi_{1}(s)\right) d s} \varphi_{2}(\tau) d \tau \\
\leq & \bar{\varphi}_{2}\left(T_{2}\right) \int_{0}^{T_{2}} e^{\int_{0}^{\tau}\left(\underline{\gamma} \omega^{2}(s)-\varphi_{1}(s)\right) d s} d \tau e^{\int_{0}^{t}\left(-\underline{\gamma} \omega^{2}(s)+\varphi_{1}(s)\right) d s} \\
& +\eta \int_{T_{2}}^{t} e^{\int_{\tau}^{t}\left(-\underline{\gamma} \omega^{2}(s)+\varphi_{1}(s)\right) d s} d \tau \\
\leq & \zeta+\eta \int_{T_{2}}^{t} e^{\int_{\tau}^{t}\left(-\underline{\gamma} \omega^{2}(s)+\frac{\gamma \rho^{2}}{4}\right) d s} d \tau \\
\leq & \zeta+\eta \int_{T_{2}}^{t} e^{-\underline{\gamma} \rho^{2}\left(\frac{t-\tau}{2}-\frac{1}{4} \sin 2 t+\frac{1}{4} \sin 2 \tau\right)+\frac{\gamma \rho^{2}}{4}(t-\tau)} d \tau \\
\leq & \zeta+\eta \int_{T_{2}}^{t} e^{\frac{\gamma \rho^{2}}{2}} e^{-\frac{\gamma \rho^{2}}{4}(t-\tau)} d \tau \\
\leq & \zeta+\eta \frac{4}{\underline{\gamma} \rho^{2}} e^{\frac{\gamma \rho^{2}}{2}}
\end{aligned}
$$

where $\zeta=\psi e^{\int_{0}^{t}\left(-\underline{\gamma} \omega^{2}(s)+\varphi_{1}(s)\right) d s}$ with $\psi=$ $\bar{\varphi}_{2}\left(T_{2}\right) \int_{0}^{T_{2}} e^{\int_{0}^{\tau}\left(\underline{\gamma} \omega^{2}(s)-\varphi_{1}(s)\right) d s} d \tau$. Due to boundedness of $\psi$, thus $\lim _{t \rightarrow \infty} \zeta(t)=0$. Furthermore $\forall \varepsilon>0$, there exists $T_{3}>0$ such that $\zeta(t) \leq \frac{\varepsilon}{2}$ for all $t \geq T_{3}$. Choose $\eta=\frac{\gamma \rho^{2}}{8} e^{-\frac{\gamma \rho^{2}}{2}} \varepsilon$, we have

$\int_{0}^{t} e^{\int_{\tau}^{t}\left(-\underline{\gamma} \omega^{2}(s)+\varphi_{1}(s)\right) d s} \varphi_{2}(\tau) d \tau \leq \varepsilon, \forall t \geq \max \left\{T_{2}, T_{3}\right\}$

which implies $\lim _{t \rightarrow \infty} \int_{0}^{t} e^{\int_{\tau}^{t}\left(-\underline{\gamma} \omega^{2}(s)+\varphi_{1}(s)\right) d s} \varphi_{2}(\tau) d \tau$ $=0$.

Therefore, it can be concluded that the right-side of inequality (9) will converge to zero as $t \rightarrow \infty$. Consequently, $\sigma(t)$ is bounded and tends to zero asymptotically, which also suggests that $V_{1}, z_{i 2}, z_{i 3}$ are bounded and converge to zero asymptotically. This completes the proof.

Remark 4 It should be noted that the proof of Lemma 3 is different from that in Lemma 6 of [24] and Lemma 2 of [25]. The requirements for the convergence of $\varphi_{1}, \varphi_{2}$ must be exponential in [24] and [25], which are relaxed to be asymptotical here, and the proof here is much more rigorous.

Lemma 4 If $u_{i 1}-\omega, u_{j 1}-\omega, z_{i 4}, z_{j 4}, z_{i 5}, z_{j 5}$ are bounded and $u_{i 1}-\omega, u_{j 1}-\omega, z_{i 4}-z_{j 4}, z_{i 5}-z_{j 5}$ asymptotically converge to zero for $1 \leq i \neq j \leq N$, then $z_{i 2}, z_{i 3}, z_{j 2}, z_{j 3}$ are bounded and $z_{i 2}-z_{j 2}$ and $z_{i 3}-z_{j 3}$ converge to zero asymptotically.
Proof First, we will prove that $z_{i 2}$ and $z_{i 3}$ are bounded for $i=1, \ldots, N$. By Eq. (4) and using means of variation of constants and initial integral methods, we have

$$
\begin{aligned}
z_{i 2}(t)= & e^{-\int_{0}^{t} g_{i 11}(s) d s}\left(z_{i 2}(0)\right. \\
& \left.+\int_{0}^{t} g_{i 12}(\sigma) e^{\int_{0}^{\sigma} g_{i 11}(s) d s} d \sigma\right), \\
z_{i 3}(t)= & e^{-\int_{0}^{t} g_{i 21}(s) d s}\left(z_{i 2}(0)\right. \\
& \left.+\int_{0}^{t} g_{i 22}(\sigma) e^{\int_{0}^{\sigma} g_{i 21}(s) d s} d \sigma\right),
\end{aligned}
$$

where

$$
\begin{aligned}
& g_{i 11}=\gamma_{1} \omega^{2}+\left(u_{i 1}-\omega\right) \gamma_{1} \omega, g_{i 12}=u_{i 1} z_{i 4}, \\
& g_{i 21}=\gamma_{2} \omega^{2}+\left(u_{i 1}-\omega\right) \gamma_{2} \omega, g_{i 22}=u_{i 1} z_{i 5}
\end{aligned}
$$

Since $u_{i 1}-\omega, \omega, z_{i 4}, z_{i 5}$ are all bounded, thus $g_{i 11}, g_{i 12}, g_{i 21}, g_{i 22}$ are bounded. Furthermore, it can be proved that $z_{i 2}$ and $z_{i 3}$ are bounded by Eq. 14 .

Let $e_{i j 2}=z_{i 2}-z_{j 2}$ for $1 \leq i \neq j \leq N$, we have

$$
\begin{aligned}
\dot{e}_{i j 2}= & -\gamma_{1} z_{i 2} \omega^{2}+\omega z_{i 4}+\left(u_{i 1}-\omega\right)\left(z_{i 4}-\gamma_{1} \omega z_{i 2}\right) \\
& +\gamma_{1} z_{j 2} \omega^{2}-\omega z_{j 4}-\left(u_{j 1}-\omega\right)\left(z_{j 4}-\gamma_{1} \omega z_{j 2}\right) \\
= & -\gamma_{1} \omega^{2} e_{i j 2}+\varphi_{i j 1}(t)
\end{aligned}
$$

where $\varphi_{i j 1}(t)=\omega\left(z_{i 4}-z_{j 4}\right)+\left(u_{i 1}-\omega\right)\left(z_{i 4}-\right.$ $\left.\gamma_{1} \omega z_{i 2}\right)-\left(u_{j 1}-\omega\right)\left(z_{j 4}-\gamma_{1} \omega z_{j 2}\right)$. Since $z_{i 4}-z_{j 4}$ and $u_{i 1}-\omega$ asymptotically converge to zero, and $\omega$, $z_{i 2}, z_{i 4}, z_{j 2}, z_{j 4}$ are bounded, thus $\varphi_{i j 1}(t)$ converge to zero asymptotically. Choose the following Lyapunov function

$V_{2}=\frac{1}{2} e_{i j}^{2}$.

Using the mimicking argument as the proof of Lemma 3 , it can be easily proved that $\lim _{t \rightarrow \infty} e_{i j 2}(t)=0$, namely, $z_{i 2}-z_{j 2}$ converges to zero asymptotically for $1 \leq i \neq j \leq N$. Also, with the similar technique, the conclusion that $z_{i 3}-z_{j 3}$ asymptotically converges to zero can be given.

\section{Controller Design and Stability Analysis}

\subsection{Closed-loop System Stability}

In this subsection, we will design the distributed control input $u_{i}$ for the $i$ th system (4) using $z_{i}$ and the relative state $z_{l}$ of its neighbors for $l \in N_{i}$ such that 
$z_{i}$ is bounded and $\lim _{t \rightarrow \infty}\left(z_{i}(t)-z_{j}(t)\right)=0$ for $1 \leq i \neq j \leq N$. The structure of system (4) suggests $z_{i 1}, z_{i 4}, z_{i 5}$ can be directly controlled via $u_{i 1}, u_{i 2}, u_{i 3}$. Now we are ready to choose the distributed controller $u_{i}$ as

$$
\begin{aligned}
u_{i 1}= & -\sum_{j=1}^{N} a_{i j}\left(z_{i 1}-z_{j 1}\right)+\omega \\
u_{i 2}= & -\sum_{j=1}^{N} a_{i j}\left(z_{i 4}-z_{j 4}\right)-\gamma_{1} \dot{\omega} z_{i 2}-\gamma_{1} \omega u_{i 1} z_{i 4} \\
& +\gamma_{1}^{2} \omega^{2} u_{i 1} z_{i 2}, \\
u_{i 3}= & -\sum_{j=1}^{N} a_{i j}\left(z_{i 5}-z_{j 5}\right)-\gamma_{2} \dot{\omega} z_{i 3}-\gamma_{2} \omega u_{i 1} z_{i 5} \\
& +\gamma_{2}^{2} \omega^{2} u_{i 1} z_{i 3} .
\end{aligned}
$$

Remark 5 The first term of Eq. 17 is a weighted sum of the relative state information between system $i$ and its neighbors. And the terms $\omega,-\gamma_{1} \dot{\omega} z_{i 2}-$ $\gamma_{1} \omega u_{i 1} z_{i 4}+\gamma_{1}^{2} \omega^{2} u_{i 1} z_{i 2},-\gamma_{2} \dot{\omega} z_{i 3}-\gamma_{2} \omega u_{i 1} z_{i 5}+$ $\gamma_{2}^{2} \omega^{2} u_{i 1} z_{i 3}$ are the canceling terms, which are designed to cancel the extra parts.

Substituting control input (17) into system (4), we can get the following closed-loop error system for $z_{i 1}, z_{i 4}, z_{i 5}$

$$
\begin{aligned}
& \dot{z}_{i 1}=-\sum_{j=1}^{N} a_{i j}\left(z_{i 1}-z_{j 1}\right), \\
& \dot{z}_{i 4}=-\sum_{j=1}^{N} a_{i j}\left(z_{i 4}-z_{j 4}\right), \\
& \dot{z}_{i 5}=-\sum_{j=1}^{N} a_{i j}\left(z_{i 5}-z_{j 5}\right) .
\end{aligned}
$$

Theorem 1 Consider the closed-loop system consisting of $N$ transformed systems (4) satisfying Assumption 1, the proposed distributed controller (17). Then the state $z_{i}$ of the ith transformed system (4) in the closed-loop system is bounded and $\lim _{t \rightarrow \infty}\left(z_{i}(t)-\right.$ $\left.z_{j}(t)\right)=0$ for $1 \leq i \neq j \leq N$.

Proof By Eq. 18, we have

$\dot{Z}_{1}=-L Z_{1}, \dot{Z}_{4}=-L Z_{4}, \dot{Z}_{5}=-L Z_{5}$,

where $Z_{q}=\left[z_{1 q}, z_{2 q}, \ldots, z_{N q}\right]$ for $q=1,4,5$, and $L$ is the Laplacian matrix of $\mathcal{G}$. Therefore

$$
Z_{1}=e^{-L t} Z_{1}(0), Z_{4}=e^{-L t} Z_{4}(0), Z_{5}=e^{-L t} Z_{5}(0) \text {. }
$$

By Lemma 1, we have

$$
\begin{aligned}
& \lim _{t \rightarrow \infty} Z_{1}(t)=\underline{1} w^{T} Z_{1}(0)=: c_{1} \underline{1}, \\
& \lim _{t \rightarrow \infty} Z_{4}(t)=\underline{1} w^{T} Z_{4}(0)=: c_{4} \underline{1}, \\
& \lim _{t \rightarrow \infty} Z_{5}(t)=\underline{1} w^{T} Z_{5}(0)=: c_{5} \underline{1} .
\end{aligned}
$$

It is apparent that $\lim _{t \rightarrow \infty}\left(z_{1 i}(t)-z_{1 j}(t)\right)=$ $0, \lim _{t \rightarrow \infty}\left(z_{4 i}(t)-z_{4 j}(t)\right)=0, \lim _{t \rightarrow \infty}\left(z_{5 i}(t)-\right.$ $\left.z_{5 j}(t)\right)=0$ for $1 \leq i \neq j \leq N$.

By utilizing Eqs. 17 and 20, we can prove that $u_{l 1}-\omega$ is bounded and converges to zero asymptotically for $l=1, \ldots, N$. Then according to Lemma 4, we have that $z_{l 2}$ and $z_{l 3}$ are bounded. In addition, the conclusion that $z_{i 2}-z_{j 2}$ and $z_{i 3}-z_{j 3}$ converge to zero asymptotically for $1 \leq i \neq j \leq N$ can also be given by Lemma 4 , namely, $\lim _{t \rightarrow \infty}\left(z_{l 2}(t)-\right.$ $\left.c_{2}(t)\right)=0, \lim _{t \rightarrow \infty}\left(z_{l 3}(t)-c_{3}(t)\right)=0$ for $l=$ $1, \ldots, N$, where $c_{2}(t)$ and $c_{3}(t)$ are unknown but bounded functions.

Remark 6 A distributed control law for system (4) is given by Eq. 17. Control law (17) can make $z_{l}$ for $l=$ $1, \ldots, N$ converge to $c(t)$ asymptotically with $c(t)=$ $\left[c_{1}, c_{2}, c_{3}, c_{4}, c_{5}\right]^{T}$. By Eq. 3, it is easy to prove that

$\lim _{t \rightarrow \infty} \bar{q}_{i}(t)-\bar{q}_{j}(t)=0,1 \leq i \neq j \leq N$

where $\bar{q}_{l}=\left[x_{l}, y_{l}, \theta_{l}\right]^{T}$ for $l=1, \ldots, N$.

The following theorem shows that we can make $z_{i}$ converge to zero. We redesign the distributed controller $u_{i}$ as

$$
\begin{aligned}
u_{i 1}= & -\sum_{j=1}^{N} a_{i j}\left(z_{i 1}-z_{j 1}\right)-p_{i} z_{i 1}+\omega, \\
u_{i 2}= & -\sum_{j=1}^{N} a_{i j}\left(z_{i 4}-z_{j 4}\right)-q_{i} z_{i 4}-\gamma_{1} \dot{\omega} z_{i 2} \\
& -\gamma_{1} \omega u_{i 1} z_{i 4}+\gamma_{1}^{2} \omega^{2} u_{i 1} z_{i 2}, \\
u_{i 3}= & -\sum_{j=1}^{N} a_{i j}\left(z_{i 5}-z_{j 5}\right)-k_{i} z_{i 5}-\gamma_{2} \dot{\omega} z_{i 3} \\
& -\gamma_{2} \omega u_{i 1} z_{i 5}+\gamma_{2}^{2} \omega^{2} u_{i 1} z_{i 3},
\end{aligned}
$$

where $p_{i} \geq 0, q_{i} \geq 0, k_{i} \geq 0$, and $\sum_{i=1}^{N} p_{i}>0$, $\sum_{i=1}^{N} q_{i}>0, \sum_{i=1}^{N} k_{i}>0$.

Remark 7 These terms $p_{i} z_{i 1}, q_{i} z_{i 4}, k_{i} z_{i 5}$ in Eq. 22 can also be considered as relative information between robot $i$ and a virtual robot with its state being zero.

Theorem 2 Consider the closed-loop system consisting of $N$ transformed systems (4) satisfying Assumption 1 , the proposed distributed controller (22) with the parameters satisfying $p_{i} \geq 0, q_{i} \geq 0, k_{i} \geq 0$, and $\sum_{i=1}^{N} p_{i}>0, \sum_{i=1}^{N} q_{i}>0, \sum_{i=1}^{N} k_{i}>0$. Then the state $z_{i}$ of the ith transformed system (4) in the closed-loop system is bounded and converges to zero asymptotically, i.e., $\lim _{t \rightarrow \infty} z_{i}(t)=0$ for $i=$ $1, \ldots, N$. 
Proof With the distributed controller $u_{i}$ defined in Eq. 22, we have

$$
\begin{aligned}
& \dot{z}_{i 1}=-\sum_{j=1}^{N} a_{i j}\left(z_{i 1}-z_{j 1}\right)-p_{i} z_{i 1}, \\
& \dot{z}_{i 4}=-\sum_{j=1}^{N} a_{i j}\left(z_{i 4}-z_{j 4}\right)-q_{i} z_{i 4}, \\
& \dot{z}_{i 5}=-\sum_{j=1}^{N} a_{i j}\left(z_{i 5}-z_{j 5}\right)-k_{i} z_{i 5} .
\end{aligned}
$$

Choose the Lyapunov function

$$
V=\frac{1}{2} \sum_{i=1}^{N}\left(z_{i 1}^{2}+z_{i 4}^{2}+z_{i 5}^{2}\right) \text {. }
$$

Differentiating $V$ along the solutions of Eq. 23 yields

$$
\begin{aligned}
\dot{V}= & -\frac{1}{2} Z_{1}^{T}\left(L^{T}+L\right) Z_{1}-\frac{1}{2} Z_{4}^{T}\left(L^{T}+L\right) Z_{4} \\
& -\frac{1}{2} Z_{5}^{T}\left(L^{T}+L\right) Z_{5} \\
& -\sum_{i=1}^{N}\left(p_{i} z_{i 1}^{2}+q_{i} z_{i 4}^{2}+k_{i} z_{i 5}^{2}\right),
\end{aligned}
$$

where $Z_{q}=\left[z_{1 q}, z_{2 q}, \ldots, z_{N q}\right]$ for $q=1,4,5$, and $L$ is the Laplacian matrix of $\mathcal{G}$. Since $L^{T}+L$ is positive semidefinite, $\dot{V} \leq 0$, hence that $V(t)$ is bounded and $z_{i 1}, z_{i 4}, z_{i 5}$ are bounded. According to the definition (24), Barbalat's Lemma [36] can be employed to prove that that $\lim _{t \rightarrow \infty} \dot{V}(t)=0$. Thus we obtain,

$$
\begin{gathered}
\lim _{t \rightarrow \infty} p_{l} z_{l 1}^{2}, q_{l} z_{l 4}^{2}, k_{l} z_{l 5}^{2}=0, \quad l=1, \ldots, N \\
\lim _{t \rightarrow \infty} Z_{1}^{T}\left(L^{T}+L\right) Z_{1}=0, \\
\lim _{t \rightarrow \infty} Z_{4}^{T}\left(L^{T}+L\right) Z_{4}=0, \\
\lim _{t \rightarrow \infty} Z_{5}^{T}\left(L^{T}+L\right) Z_{5}=0 .
\end{gathered}
$$

Since there is at least one integer $m$ such that $p_{m}>$ 0 , $\lim _{t \rightarrow \infty} z_{m 1}(t)=0$. By applying Lemma 2, $\lim _{t \rightarrow \infty}\left(z_{i 1}(t)-z_{j 1}(t)\right)=0$ for $1 \leq i \neq j \leq N$. Hence, $\lim _{t \rightarrow \infty} z_{l 1}(t)=0$ for $l=1, \ldots, N$. And $\lim _{t \rightarrow \infty} z_{l 4}(t)=0, \lim _{t \rightarrow \infty} z_{l 5}(t)=0$ can also be proved in the similar argument.

By utilizing Eqs. 22 and 26, we can prove that $u_{l 1}-\omega$ is bounded and converges to zero asymptotically for $l=1, \ldots, N$. Then according to Lemma 3 , we have $z_{l 2}, z_{l 3}$ are bounded and converge to zero asymptotically. In summary, the state $z_{l}$ of the $l$ th transformed system (4) in the closed-loop system is bounded and converges to zero asymptotically, i.e., $\lim _{t \rightarrow \infty} z_{l}(t)=0$ for $l=1, \ldots, N$.

Remark 8 By Eq. 3 and $\lim _{t \rightarrow \infty} z_{l}(t)=0$, we have $\lim _{t \rightarrow \infty}\left[\theta_{l}(t)-\rho(1-\cos t)\right]=0$, which means that $\theta_{l}$ converges to a neighborhood $B d$ of the origin with radius $\rho$. And from the second equation and third equation of Eq. 3, we can also get $x_{l}, y_{l}$ are bounded and asymptotically converge to zero provided $z_{l 2}, z_{l 3}$ are bounded and converge to zero asymptotically. From the fourth equation and fifth equation of Eq. 3, it can also be proved that if $\lim _{t \rightarrow \infty} z_{l}(t)=0$, then $\lim _{t \rightarrow \infty} \beta_{l 1}(t)=k_{l 1} \pi$ and $\lim _{t \rightarrow \infty} \beta_{l 2}(t)=k_{l 2} \pi$, $k_{l 1}, k_{l 2} \in Z$. Thus, the problem of cooperatively converging to a stationary point of a group of nonholonomic systems (2) is practically solved. In addition, if $\rho$ decreases, then the $\theta_{l}$ becomes small. However, the performance of $x_{l}, y_{l}$ becomes bad, i.e., the convergence rate of $z_{l 2}, z_{l 3}$ to zero decreases. Therefore, there is a tradeoff between small $\theta_{l}$ and a large convergence rate of $x_{l}, y_{l}$ when one chooses $\rho$.

\subsection{Closed-loop System Stability with Communication Delays}

In practice, there are always time delays due to communication and other factors. In this subsection, we will consider communication delays in the control design and analysis. For simplicity, in this paper we assume that all communication delays are constant.

Assumption 2 The communication digraph $\mathcal{G}$ is bidirectional and strongly connected.

Under Assumption 2, the distributed controller is designed as

$$
\begin{aligned}
u_{i 1}(t)= & -\sum_{j=1}^{N} a_{i j}\left(z_{i 1}(t)-z_{j 1}\left(t-\tau_{i}\right)\right)+\omega(t), \\
u_{i 2}(t)= & -\sum_{j=1}^{N} a_{i j}\left(z_{i 4}(t)-z_{j 4}\left(t-\tau_{i}\right)\right)-\gamma_{1} \dot{\omega}(t) z_{i 2}(t) \\
& -\gamma_{1} \omega(t) u_{i 1}(t) z_{i 4}(t)+\gamma_{1}^{2} \omega^{2}(t) u_{i 1}(t) z_{i 2}(t), \\
u_{i 3}(t)= & -\sum_{j=1}^{N} a_{i j}\left(z_{i 5}(t)-z_{j 5}\left(t-\tau_{i}\right)\right)-\gamma_{2} \dot{\omega}(t) z_{i 3}(t) \\
& -\gamma_{2} \omega(t) u_{i 1}(t) z_{i 5}(t)+\gamma_{2}^{2} \omega^{2}(t) u_{i 1}(t) z_{i 3}(t),
\end{aligned}
$$

where communication delay $\tau_{i}(\geq 0)$ is a positive constant.

Fig. 2 The communication graph $\mathcal{G}_{1}$

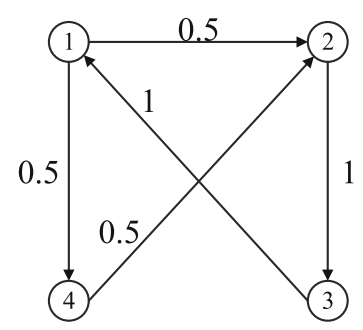


Fig. 3 Profiles of the states $z_{i 1}$ with controller (17) and controller (22)

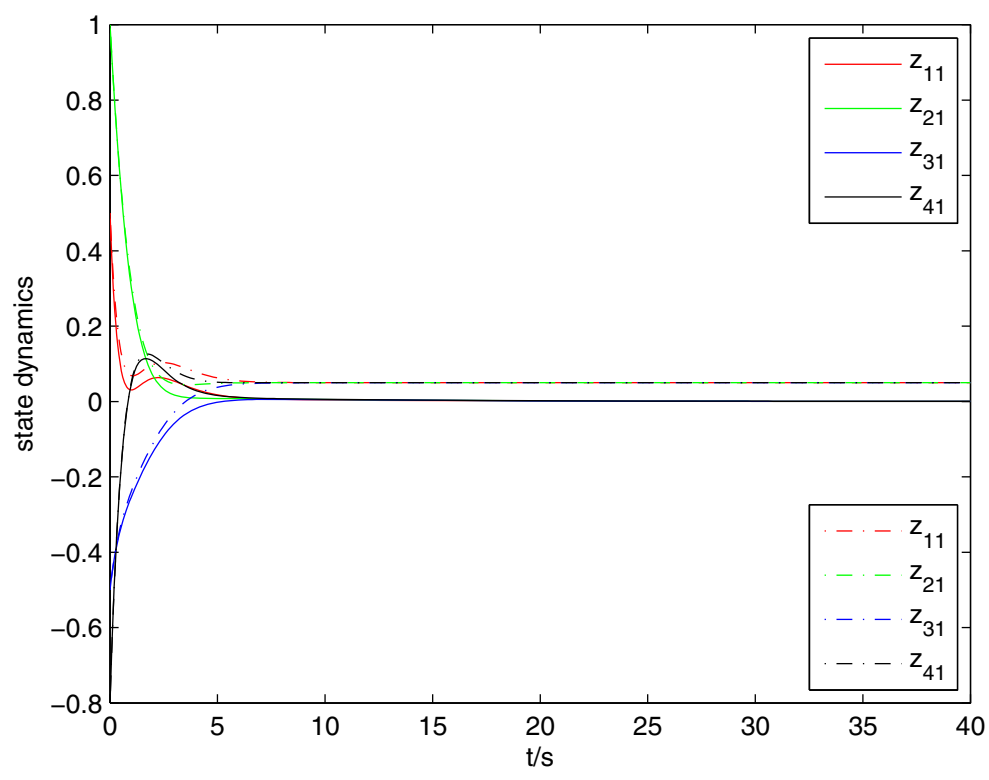

Substituting the distributed controller (27) into system (4), we can get the following closed-loop error system for $z_{i 1}, z_{i 4}, z_{i 5}$

$$
\begin{aligned}
& \dot{z}_{i 1}(t)=-\sum_{j=1}^{N} a_{i j}\left(z_{i 1}(t)-z_{j 1}\left(t-\tau_{i}\right)\right), \\
& \dot{z}_{i 4}(t)=-\sum_{j=1}^{N} a_{i j}\left(z_{i 4}(t)-z_{j 4}\left(t-\tau_{i}\right)\right), \\
& \dot{z}_{i 5}(t)=-\sum_{j=1}^{N} a_{i j}\left(z_{i 5}(t)-z_{j 5}\left(t-\tau_{i}\right)\right) .
\end{aligned}
$$

Theorem 3 Consider the closed-loop system consisting of $N$ transformed systems (4) satisfying Assumption 2, and the proposed distributed controller
(27). Then the state $z_{i}$ of the ith transformed system (4) in the closed-loop system is bounded and $\lim _{t \rightarrow \infty}\left(z_{i}(t)-z_{j}(t)\right)=0$ for $1 \leq i \neq j \leq N$.

Proof Let

$$
\begin{aligned}
V(t)= & \frac{1}{2} \sum_{i=1}^{N}\left(z_{i 1}^{2}(t)+\sum_{j=1}^{N} a_{i j} \int_{t-\tau_{i}}^{t} z_{j 1}^{2}(s) d s\right) \\
& +\frac{1}{2} \sum_{i=1}^{N}\left(z_{i 4}^{2}(t)+\sum_{j=1}^{N} a_{i j} \int_{t-\tau_{i}}^{t} z_{j 4}^{2}(s) d s\right) \\
& +\frac{1}{2} \sum_{i=1}^{N}\left(z_{i 5}^{2}(t)+\sum_{j=1}^{N} a_{i j} \int_{t-\tau_{i}}^{t} z_{j 5}^{2}(s) d s\right) .
\end{aligned}
$$

Fig. 4 Profiles of the states $z_{i 2}$ with controller (17) and controller (22)

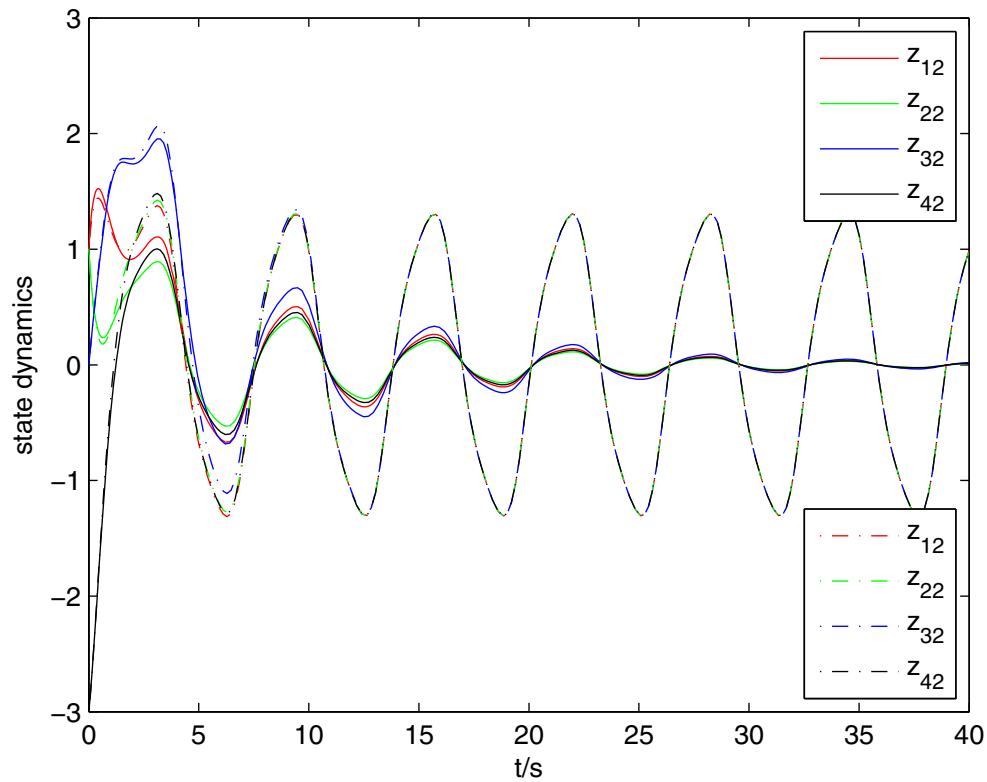


Fig. 5 Profiles of the states $z_{i 3}$ with controller (17) and controller (22)

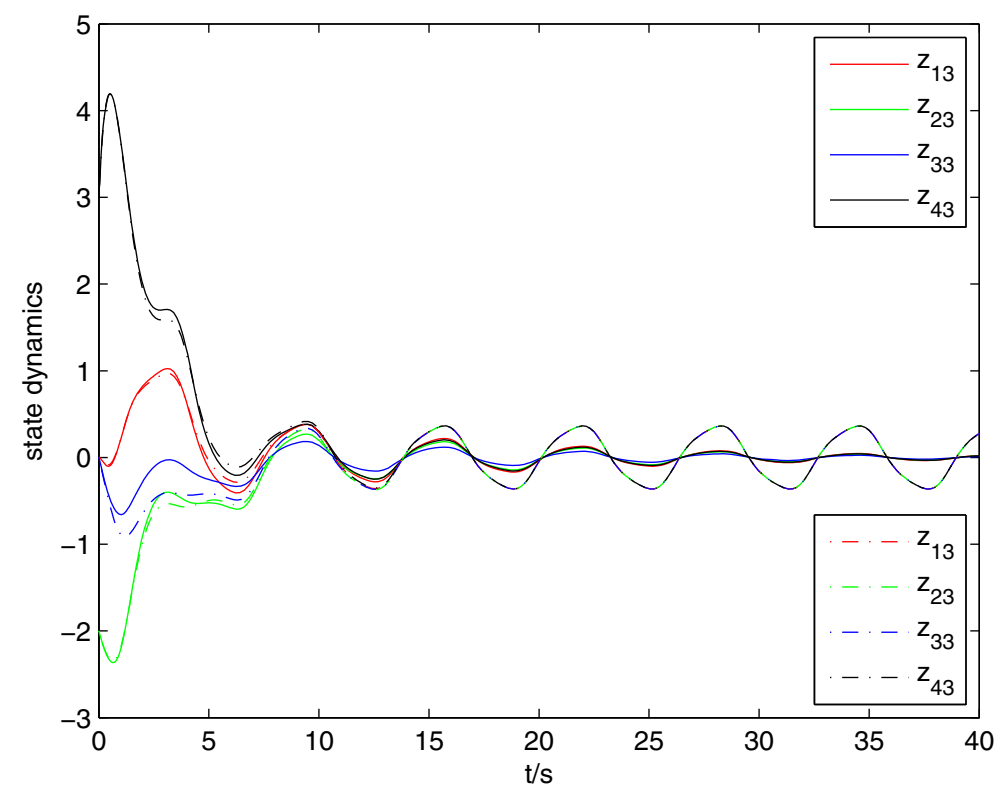

Differentiate $V$ along the solutions of Eq. 28 yields

$$
\begin{aligned}
\dot{V}(t)= & -\frac{1}{2} \sum_{i=1}^{N} \sum_{j=1}^{N} a_{i j}\left(z_{i 1}(t)-z_{j 1}\left(t-\tau_{i}\right)\right)^{2} \\
& -\frac{1}{2} \sum_{i=1}^{N} \sum_{j=1}^{N} a_{i j}\left(z_{i 4}(t)-z_{j 4}\left(t-\tau_{i}\right)\right)^{2} \\
& -\frac{1}{2} \sum_{i=1}^{N} \sum_{j=1}^{N} a_{i j}\left(z_{i 5}(t)-z_{j 5}\left(t-\tau_{i}\right)\right)^{2} \leq 0,
\end{aligned}
$$

where the fact that the communication graph $\mathcal{G}$ is bidirectional has been used. By the invariance principle
[37], $z_{l 1}, z_{l 4}$, and $z_{l 5}$ will converge to constants for $l=1, \ldots, N$. The following proof is the same as the proof in Theorem 1, but omitted here.

Remark 9 In practice, there are always time delays due to communication and other factors. In our manuscript, we take time delays into account in our design of distributed protocol and we allow the delays to be arbitrarily large. In the theorem, communication delays only appear in the neighbors states. This
Fig. 6 Profiles of the states $z_{i 4}$ with controller (17) and controller (22)

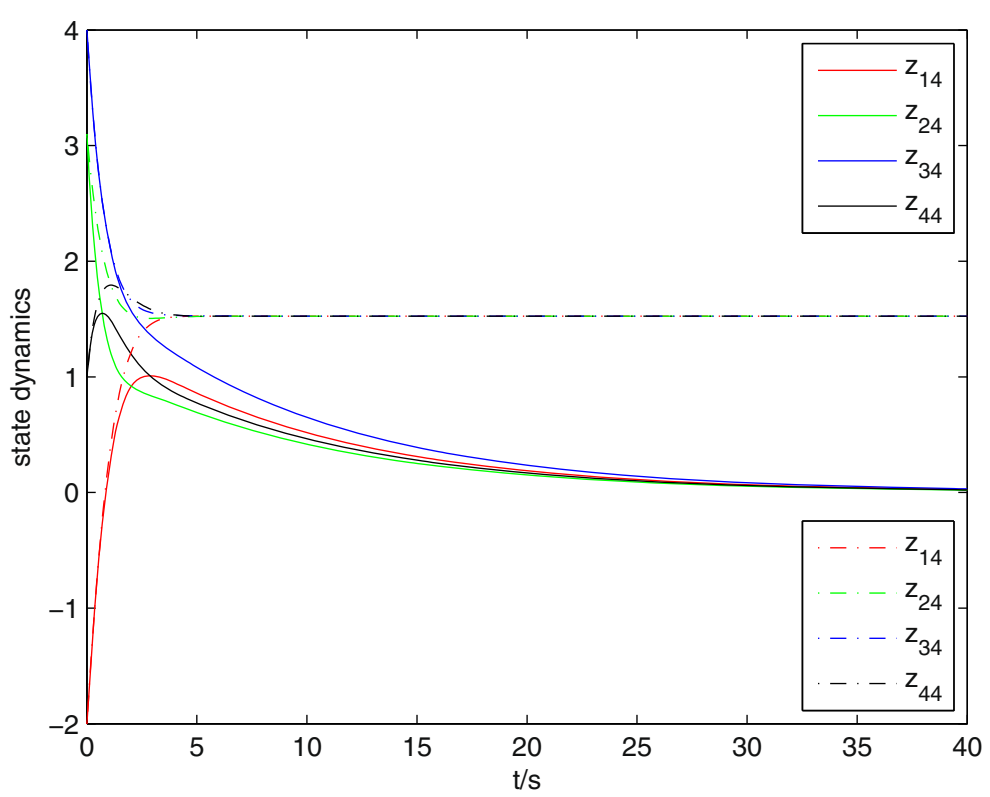


Fig. 7 Profiles of the states $z_{i 5}$ with controller (17) and controller (22)

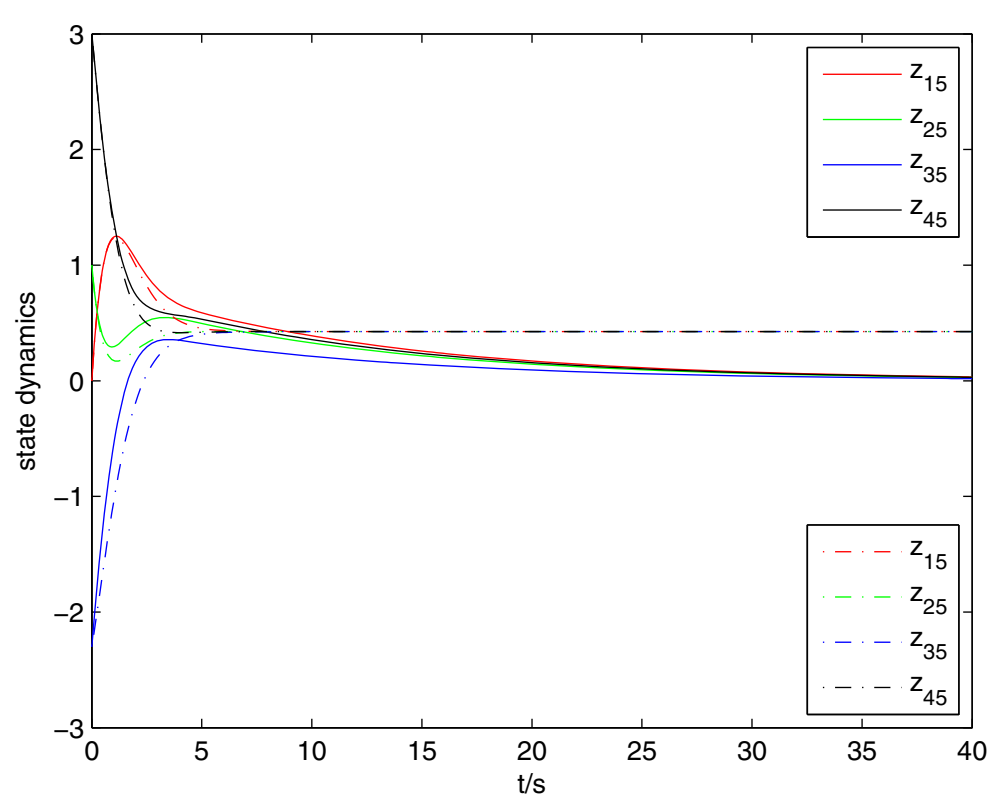

assumption is reasonable because the communication delay is the dominated delay among all other time delays. The first term of Eq. 22 can be treated as a weighted sum of the relative state information between the current states of system $i$ and the delayed state information of its neighboring. By applying the invariance principle, it is proved that our proposed cooperative control laws are still effective even existing communication delay. Assumption 2 is stronger than Assumption 1, since the existence of delays is in the communication.

Corresponding to Theorem 2, we have the following delayed version result.

Theorem 4 Consider the system consisting of $N$ transformed systems (4) satisfying Assumption 2, and use distributed controller given by

$$
\begin{aligned}
u_{i 1}(t)= & -\sum_{j=1}^{N} a_{i j}\left(z_{i 1}(t)-z_{j 1}\left(t-\tau_{i}\right)\right)-p_{i} z_{i 1}(t)+\omega(t) \\
u_{i 2}(t)= & -\sum_{j=1}^{N} a_{i j}\left(z_{i 4}(t)-z_{j 4}\left(t-\tau_{i}\right)\right)-q_{i} z_{i 4}(t)-\gamma_{1} \dot{\omega}(t) z_{i 2}(t) \\
& -\gamma_{1} \omega(t) u_{i 1}(t) z_{i 4}(t)+\gamma_{1}^{2} \omega^{2}(t) u_{i 1}(t) z_{i 2}(t) \\
u_{i 3}(t)= & -\sum_{j=1}^{N} a_{i j}\left(z_{i 5}(t)-z_{j 5}\left(t-\tau_{i}\right)\right)-k_{i} z_{i 5}(t)-\gamma_{2} \dot{\omega}(t) z_{i 3}(t) \\
& -\gamma_{2} \omega(t) u_{i 1}(t) z_{i 5}(t)+\gamma_{2}^{2} \omega^{2}(t) u_{i 1}(t) z_{i 3}(t)
\end{aligned}
$$

with the parameters satisfying $p_{i} \geq 0, q_{i} \geq 0, k_{i} \geq 0$, and $\sum_{i=1}^{N} p_{i}>0, \sum_{i=1}^{N} q_{i}>0, \sum_{i=1}^{N} k_{i}>0$, where communication delay $\tau_{i}(\geq 0)$ is a positive constant. Then the state $z_{i}$ of the ith transformed system (4) in the closed-loop system is bounded and converges to zero asymptotically, i.e., $\lim _{t \rightarrow \infty} z_{i}(t)=0$ for $i=$ $1, \ldots, N$.

Proof The proof is analogous as that of Theorem 2 and Theorem 3 and is omitted here.

\section{Extensions}

In practical applications, multiple type $(1,2)$ nonholonomic mobile robots may need to achieve a prescribed formation other than rendezvousing at a common value. It is shown that, if convergence to a common value is feasible, then other formations can also be obtained by the simple transformation.

Definition 2 The formation control problem discussed in this paper is to design a distributed controller for the $i$ th system (2), based on its state information

Fig. 8 The communication graph $\mathcal{G}_{2}$ with time-delays

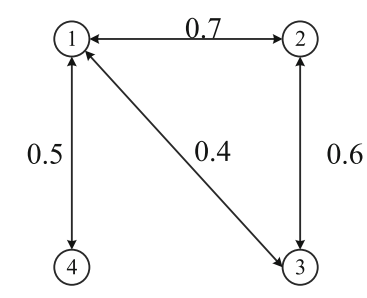


Fig. 9 Profiles of the states $z_{i 1}$ with communication delays $\tau=0.5 \mathrm{~s}$ and $\tau=2.5 \mathrm{~s}$

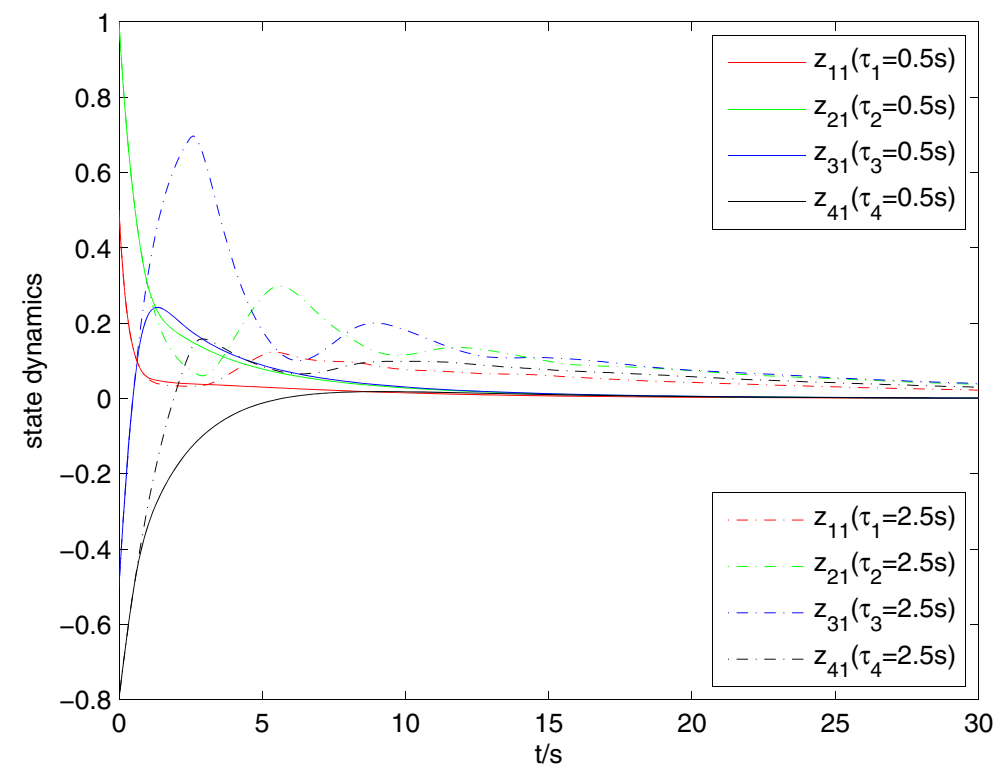

$q_{i}$ and the relative state $q_{l}$ of its neighbors for $l \in N_{i}$ such that

$\lim _{t \rightarrow \infty}\left(\left[\begin{array}{c}x_{i}-x_{j} \\ y_{i}-y_{j}\end{array}\right]-\left[\begin{array}{cc}\cos \chi & \sin \chi \\ -\sin \chi & \cos \chi\end{array}\right]\left[\begin{array}{c}p_{i x}-p_{j x} \\ p_{i y}-p_{j y}\end{array}\right]\right)=0$,
$\lim _{t \rightarrow \infty}\left(\theta_{i}(t)-\theta_{j}(t)\right)=0,1 \leq i \neq j \leq N$ $\lim _{t \rightarrow \infty} \sum_{i=1}^{N} x_{i}=\sum_{i=1}^{N} p_{i x}, \lim _{t \rightarrow \infty} \sum_{i=1}^{N} y_{i}=\sum_{i=1}^{N} p_{i y}$, $\lim _{t \rightarrow \infty} \sum_{i=1}^{N} \beta_{i 1}=k_{1} \pi, \lim _{t \rightarrow \infty} \sum_{i=1}^{N} \beta_{i 1}=k_{2} \pi, k_{1}, k_{2} \in Z$

where $\chi$ is a free variable, and $p_{i x}, p_{i y}$ are the prescribed displacements between the state value $x_{i}, y_{i}$ of robot $i$ and the system consensus value, which is
Fig. 10 Profiles of the states $z_{i 2}$ with communication delays $\tau=0.5 \mathrm{~s}$ and $\tau=2.5 \mathrm{~s}$

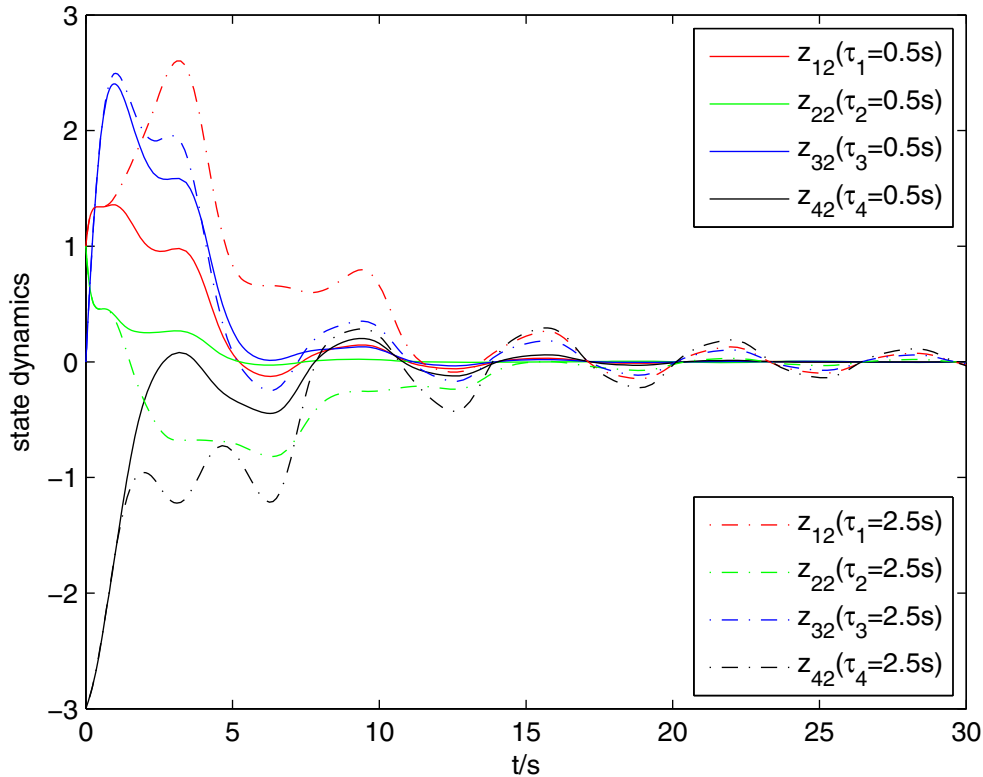


Fig. 11 Profiles of the states $z_{i 3}$ with communication delays $\tau=0.5 \mathrm{~s}$ and $\tau=2.5 \mathrm{~s}$

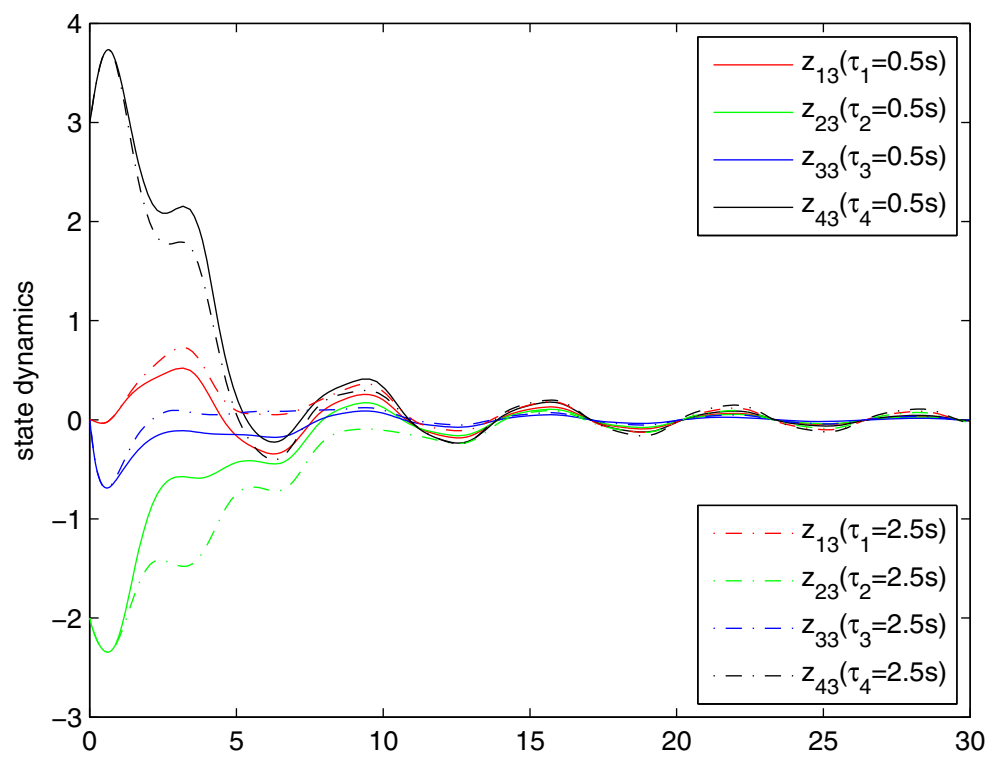

unknown and depends on robots' initial conditions and communication between robots.

Let

$$
\begin{aligned}
& \bar{z}_{i 1}=\theta_{i}-\int_{0}^{t} \omega(s) d s, \\
& \bar{z}_{i 2}=\left(x_{i}-p_{i x}\right) \cos \theta_{i}+\left(y_{i}-p_{i y}\right) \sin \theta_{i}, \\
& \bar{z}_{i 3}=\left(x_{i}-p_{i x}\right) \sin \theta_{i}-\left(y_{i}-p_{i y}\right) \cos \theta_{i}, \\
& \bar{z}_{i 4}=-\bar{z}_{i 3}-2 l_{r} \frac{\sin \beta_{i 1} \sin \beta_{i 2}}{\sin \left(\beta_{i 2}-\beta_{i 1}\right)}+\gamma_{1} \omega \bar{z}_{i 2},
\end{aligned}
$$

$$
\begin{aligned}
\bar{z}_{i 5}= & \bar{z}_{i 2}-l_{r} \frac{\sin \left(\beta_{i 1}+\beta_{i 2}\right)}{\sin \left(\beta_{i 2}-\beta_{i 1}\right)}+\gamma_{2} \omega \bar{z}_{i 3}, \\
\bar{u}_{i 1}= & v_{i 1} \sin \left(\beta_{i 2}-\beta_{i 1}\right), \\
\bar{u}_{i 2}= & -v_{i 1} \sin \left(\beta_{i 2}-\beta_{i 1}\right) \bar{z}_{i 2}+2 l_{r} v_{i 3} \frac{\sin ^{2} \beta_{i 1}}{\sin ^{2}\left(\beta_{i 2}-\beta_{i 1}\right)} \\
& -2 l_{r} v_{i 2} \frac{\sin ^{2} \beta_{i 2}}{\sin ^{2}\left(\beta_{i 2}-\beta_{i 1}\right)}+l_{r} v_{i 1} \sin \left(\beta_{i 1}+\beta_{i 2}\right), \\
\bar{u}_{i 3}= & v_{i 1} \sin \left(\beta_{i 2}-\beta_{i 1}\right) \bar{z}_{i 3}+l_{r} v_{i 3} \frac{\sin \left(2 \beta_{i 1}\right)}{\sin ^{2}\left(\beta_{i 2}-\beta_{i 1}\right)} \\
& -l_{r} v_{i 2} \frac{\sin \left(2 \beta_{i 2}\right)}{\sin ^{2}\left(\beta_{i 2}-\beta_{i 1}\right)}+2 l_{r} v_{i 1} \sin \beta_{i 1} \sin \beta_{i 2},
\end{aligned}
$$

Fig. 12 Profiles of the states $z_{i 4}$ with communication delays $\tau=0.5 \mathrm{~s}$ and $\tau=2.5 \mathrm{~s}$

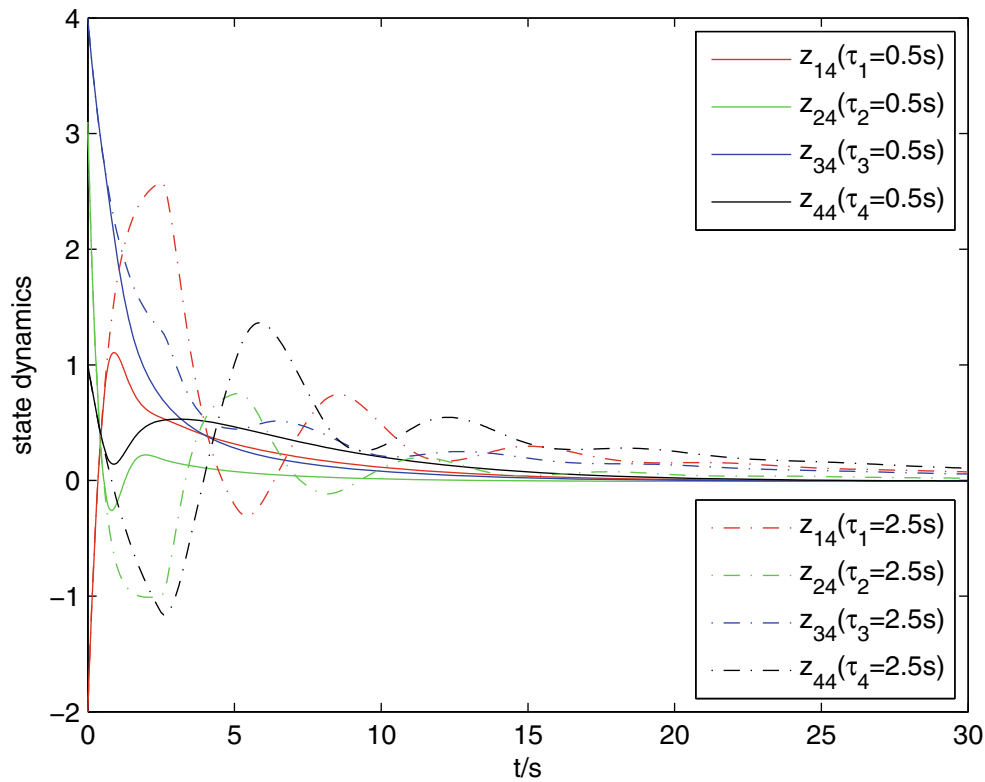


Fig. 13 Profiles of the states $z_{i 5}$ with communication delays $\tau=0.5 \mathrm{~s}$ and $\tau=2.5 \mathrm{~s}$

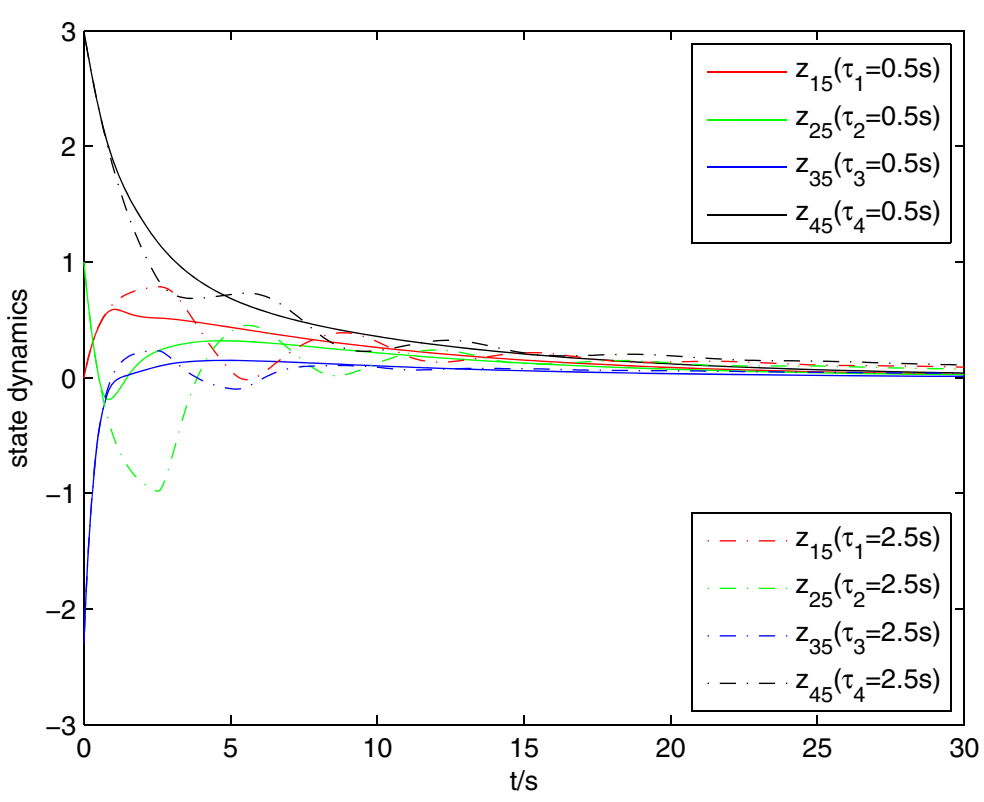

where $\omega=\rho \sin t$, and $\rho, \gamma_{1}, \gamma_{2}$ are positive constants. Taking derivative of Eq. 34, we have

$$
\begin{aligned}
& \dot{\bar{z}}_{i 1}=\bar{u}_{i 1}-\omega, \\
& \dot{\bar{z}}_{i 2}=-\gamma_{1} \bar{z}_{i 2} \omega^{2}+\omega \bar{z}_{i 4}+\left(\bar{u}_{i 1}-\omega\right)\left(\bar{z}_{i 4}-\gamma_{1} \omega \bar{z}_{i 2}\right), \\
& \dot{\bar{z}}_{i 3}=-\gamma_{2} \bar{z}_{i 3} \omega^{2}+\omega \bar{z}_{i 5}+\left(\bar{u}_{i 1}-\omega\right)\left(\bar{z}_{i 5}-\gamma_{2} \omega \bar{z}_{i 3}\right), \\
& \dot{\bar{z}}_{i 4}=\bar{u}_{i 2}+\gamma_{1} \dot{\omega} \bar{z}_{i 2}+\gamma_{1} \omega \bar{u}_{i 1} \bar{z}_{i 4}-\gamma_{1}^{2} \omega^{2} \bar{u}_{i 1} \bar{z}_{i 2}, \\
& \dot{\bar{z}}_{i 5}=\bar{u}_{i 3}+\gamma_{2} \dot{\omega} \bar{z}_{i 3}+\gamma_{2} \omega \bar{u}_{i 1} \bar{z}_{i 5}-\gamma_{2}^{2} \omega^{2} \bar{u}_{i 1} \bar{z}_{i 3} .
\end{aligned}
$$

Lemma 5 If $\lim _{t \rightarrow \infty}\left(\bar{Z}_{i}(t)-\bar{Z}_{j}(t)\right)=0$ for $1 \leq i \neq j \leq N$, then Eq. 32 holds, where $\bar{Z}_{i}(t)=\left[\bar{z}_{i 1}, \bar{z}_{i 2}, \bar{z}_{i 3}, \bar{z}_{i 4}, \bar{z}_{i 5}\right]^{T}$. Furthermore, if $\lim _{t \rightarrow \infty} \bar{Z}_{l}(t)=0$ for $l=1, \ldots, N$, then Eqs. 32 and 33 hold.

By replacing $z_{i j}$ in Eqs. 17, 22, 27, and 31 with $\bar{z}_{i j}$ for $j=1, \ldots, 5$, similar control algorithms can be obtained. By Lemma 5, the formation control problem is also solved.

\section{Simulations}

We consider some examples to illustrate the proposed design schemes and verify the established theoretical results. Consider the system (4) discussed in
Section 2.3. Let $N=4$ and the initial values of each system be

$$
\begin{aligned}
z_{1}=[0.5,1,0,-2,0]^{T}, z_{2} & =[1,1,-2,3.1,1]^{T}, \\
z_{3}=[-0.5,0,0,4,-2.3]^{T}, z_{4} & =[-0.8,-3,3,1,3]^{T} .
\end{aligned}
$$

Case 1 The communication graph $\mathcal{G}_{1}$ without communication delays is described in Fig. 2. Note that this communication graph $\mathcal{G}_{1}$ satisfies Assumption 1. The corresponding adjacency matrix $A_{1}$ is given by

$A_{1}=\left[\begin{array}{cccc}0 & 0 & 1 & 0 \\ 0.5 & 0 & 0 & 0.5 \\ 0 & 1 & 0 & 0 \\ 0.5 & 0 & 0 & 0\end{array}\right]$

Two simulations are respectively implemented for the distributed control law (17) and the distributed control law (22) with $p_{1}=0.5, q_{2}=0.5, k_{3}=0.5$ and other control parameters are all zero. We choose the parameter $\rho=1$ in local change of coordinates and feedback (3). The simulations are conducted by the Matlab "ode45" method. The trajectories of states versus time plotted using solid line and dash-dot line shown in Figs. 3, 4, 5, 6 and 7 are corresponding to the distributed controller (17) and the distributed controller (22), respectively. Note that the states do not converge 
to zero directly, but are the same as its neighbors'. It demonstrates that if $z_{i 1}, z_{i 4}, z_{i 5}$ converge to nonzero constants, then $z_{i 2}$ and $z_{i 3}$ are bounded. Furthermore, if $z_{i 1}, z_{i 4}$, and $z_{i 5}$ converge to zero asymptotically, then $z_{i 2}$ and $z_{i 3}$ also converge to zero asymptotically.

Case 2 The communication graph $\mathcal{G}_{2}$ with communication delays is described in Fig. 8. Note that this communication graph $\mathcal{G}_{2}$ satisfies Assumption 2.

The corresponding adjacency matrix $A_{2}$ is given by

$$
A_{2}=\left[\begin{array}{cccc}
0 & 0.7 & 0.4 & 0.5 \\
0.7 & 0 & 0.6 & 0 \\
0.4 & 0.6 & 0 & 0 \\
0.5 & 0 & 0 & 0
\end{array}\right]
$$

To simplify the simulation, we assume all the communication delays are common to each system, namely $\tau_{1}=\tau_{2}=\tau_{3}=\tau_{4}=\tau$. The simulation is implemented for the distributed control law (27). We choose the parameter $\rho=1$ in local change of coordinates and feedback (3), $p_{1}=1.5, q_{2}=1.5, k_{3}=1.5$ and other control parameters are all zero. In order to better analyze the influence of communication delays for the system, $\tau$ is set to be $0.5 \mathrm{~s}, 2.5 \mathrm{~s}$ in the two simulations, respectively. The simulations are performed by the Matlab "dde23" method. The trajectories of states versus time plotted using solid line and dash-dot line shown in Figs. 9, 10, 11, 12 and 13 are corresponding to the time delay $\tau=0.5 \mathrm{~s}$ and $\tau=2.5 \mathrm{~s}$, respectively. Figs. 9-13 verify the fact that the states of every system (4) converge to zero asymptotically even with communication delays. It also indicates that the asymptotical convergence of the states can also be achieved for large constant delays. However, the cooperative performance is bad if communication delays are large.

\section{Conclusion}

In this paper, the distributed cooperative control problem has been investigated for type $(1,2)$ nonholonomic mobile robots. Four distributed controllers are designed to ensure that the state of the transformed system converges to the common value or zero asymptotically with and without considering communication delays. Extension is also provided to extend the proposed schemes to the case, where the nonholonomic mobile robot needs to form a stable formation other than rendezvousing at a common value. The stability of the proposed methods is proved rigorously. Simulation results confirm the effectiveness of the proposed methods. It is our future work to solve the consensus problem for multiple nonholonomic mobile robots based on visual servoing.

Acknowledgments This paper was partially supported by The Scientific Innovation program (13ZZ115), National Natural Science Foundation (61374040, 61203143), Hujiang Foundation of China (C14002), Graduate Innovation program of Shanghai (54-13-302-102).

Open Access This article is distributed under the terms of the Creative Commons Attribution 4.0 International License (http:// creativecommons.org/licenses/by/4.0/), which permits unrestricted use, distribution, and reproduction in any medium, provided you give appropriate credit to the original author(s) and the source, provide a link to the Creative Commons license, and indicate if changes were made.

\section{References}

1. Balch, T., Arkin, R.C.: Behavior-based formation control for multirobot teams. IEEE Trans. Robot. Autom. 14(6), 926-939 (1998)

2. Lin, Z., Francis, B., Maggiore, M.: Necessary and sufficient graphical conditions for formation control of unicycles. IEEE Trans. Autom. Control 50(1), 121-127 (2005)

3. Cortés, J., Bullo, F.: Coordination and geometric optimization via distributed dynamical systems. SIAM J. Control. Optim. 44(5), 1543-1574 (2005)

4. Yu, W., Chen, G., Cao, M.: Distributed leader-follower flocking control for multi-agent dynamical systems with time-varying velocities. Syst. Control Lett. 59(9), 543-552 (2010)

5. Su, H., Wang, X., Lin, Z.: Flocking of multi-agents with a virtual leader. IEEE Trans. Autom. Control 54(2), 293-307 (2009)

6. Olfati-Saber, R., Murray, R.M.: Consensus problems in networks of agents with switching topology and time-delays. IEEE Trans. Autom. Control. 49(9), 1520-1533 (2004)

7. Ren, W., Beard, R.W.: Consensus seeking in multiagent systems under dynamically changing interaction topologies. IEEE Trans. Autom. Control. 50(5), 655-661 (2005)

8. Hou, Z.G., Cheng, L., Tan, M.: Decentralized robust adaptive control for the multiagent system consensus problem using neural networks. IEEE Transactions on Systems, Man, and Cybernetics. Part B: Cybern. 39(3), 636-647 (2009) 
9. Ren, W.: Information consensus in multivehicle cooperative control. IEEE Control. Syst. Mag. 27(2), 71-82 (2007)

10. Wang, W., Huang, J., Wen, C., Fan, H.: Distributed adaptive control for consensus tracking with application to formation control of nonholonomic mobile robots. Autom. 50(4), 1254-1263 (2014)

11. Zhang, H., Lewis, F.L.: Adaptive cooperative tracking control of higher-order nonlinear systems with unknown dynamics. Autom. 48(7), 1432-1439 (2012)

12. Hu, G.: Robust consensus tracking for an integratortype multi-agent system with disturbances and unmodelled dynamics. Int. J. Control. 84(1), 1-8 (2011)

13. Das, A., Lewis, F.L.: Distributed adaptive control for synchronization of unknown nonlinear networked systems. Autom. 46(12), 2014-2021 (2010)

14. Su, H., Chen, G., Wang, X., et al.: Adaptive secondorder consensus of networked mobile agents with nonlinear dynamics. Autom. 47(2), 368-375 (2011)

15. Yu, W., Chen, G., Cao, M.: Some necessary and sufficient conditions for second-order consensus in multiagent dynamical systems. Autom. 46(6), 1089-1095 (2010)

16. Wang, Z., Zhang, W., Guo, Y.: Adaptive output consensus tracking of uncertain multi-agent systems. In: Proceedings of the 2011 American Control Conference, pp. 3387-3392 (2011)

17. Tian, Y.P., Zhang, Y.: High-order consensus of heterogeneous multi-agent systems with unknown communication delays. Autom. 48(6), 1205-1212 (2012)

18. Rrockett, R.W.: Asymptotic stability and feedback stabilization. Defense Technical Information Center (1983)

19. Jiang, Z.P., Nijmeijer, H.: A recursive technique for tracking control of nonholonomic systems in chained form. IEEE Trans. Autom. Control 44(2), 265-279 (1999)

20. Jiang, Z., Nijmeijer, H.: Tracking control of mobile robots: a case study in backstepping. Autom. 33(7), 1393-1399 (1997)

21. Wang, C.: Semiglobal practical stabilization of nonholonomic wheeled mobile robots with saturated inputs. Autom. 44(3), 816-822 (2008)

22. Sordalen, O.J., Egeland, O.: Exponential stabilization of nonholonomic chained systems. IEEE Trans. Autom. Control 40(1), 35-49 (1995)

23. Tian, Y.P., Li, S.: Exponential stabilization of nonholonomic dynamic systems by smooth time-varying control. Autom. 38(7), 1139-1146 (2002)

24. Dong, W., Farrell, J.A.: Cooperative control of multiple nonholonomic mobile agents. IEEE Trans. Autom. Control 53(6), 1434-1448 (2008)

25. Dong, W., Farrell, J.A.: Decentralized cooperative control of multiple nonholonomic dynamic systems with uncertainty. Autom. 45(3), 706-710 (2009)

26. Liu, T., Jiang, Z.P.: Distributed formation control of nonholonomic mobile robots without global position measurements. Autom. 49(2), 592-600 (2013)

27. Dong, W.: Distributed tracking control of networked chained systems. Int. J. Control. 86(12), 2159-2174 (2013)
28. Cao, K.C., Jiang, B., Yue, D.: Consensus of multiple nonholonomic chained form systems. Syst. Control Lett. 72, 61-70 (2014)

29. Campion, G., Bastin, G., D’Andréa-Novel, B.: Structural Properties and Classification of Kinematic and Dynamic Models of Wheeled Mobile Robots. IEEE Trans. Robot. Autom. 12(1), 47-62 (1996)

30. Murray, R.M., Sastry, S.S.: Nonholonomic motion planning: Steering using sinusoids. IEEE Trans. Autom. Control. 38(5), 700-716 (1993)

31. Bushnell, L.G., Tilbury, D.M., Sastry, S.S.: Steering threeinput nonholonomic systems: the fire truck example. The J. Robot. Res. 14(4), 366-381 (1995)

32. Leroquais, W., D'Andréa-Novel, B.: Transformation of the kinematic models of restricted mobility wheeled mobile robots with a single platform into chain forms. In: Proceedings of the 34th IEEE Conference on Decision and Control, vol. 4, pp. 3811-3816 (1995)

33. Dong, W.: Flocking of multiple mobile robots based on backstepping. IEEE Transactions on Systems, Man, and Cybernetics. Part B: Cybern. 41(2), 414-424 (2011)

34. Ou, M., Du, H., Li, S.: Finite-time formation control of multiple nonholonomic mobile robots. Int. J. Robust and Nonlinear Control 24(1), 140-165 (2014)

35. Feng, J., Wen, G.X.: Adaptive NN consensus tracking control of a class of nonlinear multi-agent systems. Neurocomputing 151, 288-295 (2015)

36. Khalil, H.K., Grizzle, J.W.: Nonlinear systems. Upper Saddle River, Prentice hall (2002)

37. Haddock, J.R., Terjeki, J.: Liapunov-Razumikhin functions and an invariance principle for functional differential equations. J. Differ. Equat. 48(1), 95-122 (1983)

Gang Wang received the B.Sc. degree in information and computing science from University of Shanghai for Science and Technology, Shanghai, China, in 2012, where he is currently pursuing the Ph.D. degree in systems analysis and integration. His current research interests include distributed control of nonlinear systems, adaptive control and robotics.

Chaoli Wang received his Ph.D. degree in Control Theory and Engineering at Beijing Univ. of Aero. and Astro. in China in 1999, following his M.Sc. degree and B.Sc. degree in Applied Maths at Lanzhou University in Lan Zhou, China, respectively in 1992 and 1986. Currently, he is a Professor with the Department of Electrical Engineering at the University of Shanghai for Science and Technology. Wangs research interests include nonlinear control, robust control, robot dynamic and control, visual servoing feedback control and pattern identification.

Qinghui Du received the Ph.D. degree from the Department of Control Science and Engineering at University of Shanghai for Science and Technology, China, in 2015. She is currently a Lecturer with the Department of Mathematics, Luoyang Normal University, Luoyang, China. Her main research interests include the control of stochastic nonholonomic systems. 
Lin Li received B.E. degree in automation from Qufu Normal University, Qufu, China, in 2004, and the Ph.D. degree in control theory and control engineering from Beihang University, Beijing, China, in 2010. She is currently with University of Shanghai for Science and Technology as an Associate Professor. Her current research interests include robust control and filtering, adaptive control and the cooperative control of multi-agent systems.
Wenjie Dong received his MS degree in automatic control from Beijing University of Aeronautics and Astronautics in 1996 and $\mathrm{PhD}$ degree in electrical engineering from the University of California, Riverside, in 2009. He is an Associate Professor in the Department of Electrical Engineering, the University of Texas Rio Grande Valley, Edinburg, TX. His research interests include robot control, non-linear system control, mobile sensor networks, robot vision, embedded systems, autonomous systems, etc. 